\title{
The secrets of Segway revealed to students: revisiting the inverted pendulum
}

\author{
T-P Azevedo Perdicoúlis \\ Dep. das Engenharias, ECT, UTAD \\ ISR-Coimbra \\ Coimbra, Portugal \\ tazevedo@utad.pt
}

\author{
P. Lopes dos Santos \\ Faculdade de Engenharia da Universidade do Porto \\ Universidade do Porto \\ Porto, Portugal \\ pjsantos@fe.up.pt
}

\begin{abstract}
This article revisits the inverted pendulum-in particular, analyses a simplified model of a Segway, with a view to exploring its capabilities in Control Systems Engineering education. The integration between the theoretic and practical side is achieved through simulation, and in particular by using MathWorks software. We also present a structure for the work to be done in the Laboratory class and propose a solution for the problem.
\end{abstract}

Index Terms-Cascade control, control systems engineering education, inverted-pendulum, PID control, root-locus, segway, SIMO systems, stabilisation.

\section{INTRODUCTION}

The Segway personal transportation device is at its heart a twowheeled inverted pendulum, designed both to balance upright and to translate the rider and device to a new position. The pendulum is not directly actuated; it is free to swing around its point of marginal stability in the vertical position. It can only be controlled by exerting a force on the wheels, and the reaction force is used to reposition the pendulum in the vertical.

Due to the fame of this transportation device, two wheeled inverted balancing robots have become popular projects for students and hobbysts. Here we look at a simplified model, that is a robot with two wheels connected by an axis that moves in a straight line on a level surface, and with an inverted pendulum mounted on the wheels' axis. Despite its simplicity, this is still a typical nonlinear system with an unstable equilibrium when the rob is in the vertical position, whose principal control task is to balance the pendulum in the upright position, and then drive the robot in the $x$-axis direction.

The Segway is a SIMO system, where the motors' torque is the input and the angle of the pendulum, $\Theta_{2}$, and the angle of the wheels as they turn, $\Theta_{1}$, are the outputs. See Fig. 1.

We find this to be a very good example to motivate students in an introductory course, both in classical as well as in modern control. Many times the subjects are taught in the classroom as a sequence of tools without giving the students neither any inside about the practical problems where the concepts could be used nor a holistic approach, where theory is combined with simulation and implementation, the praxis vindicated by Aström and others [3], [6]. The simplified Segway is a very good starting point to integrate these two concepts in the classroom since it is a very popular gadget and also a very rich problem from the control theory teaching point of view.

The problem is being revisited here, because we find it a powerful case study to illustrate a course in control theory. It is able to integrate (and cover) pretty much all the main topics of classical control, such as modelling, model linearisation, calculation of transfer functions, poles and zeros; stability, design of controls; analysis of stability margins using Bode diagrams and/or Nyquist plots; assess the impulse and step response of a system; tracing root-locus and using it to design a compensator [6], [13]. Last, but not least important, the physical feasibility of the system should also be evaluated. Furthermore, it is a useful problem to move from classical control to a state-space approach, were we can study directly the SIMO system and see that the notion of state feedback is important, and sometimes even necessary, to control some kind of systems.

Besides all of this, the Segway is still a fairly simple problem, and a small system, that works autonomously and can be very useful in developing intuition. Physical intuition should play a major role in teaching and learning on Control Systems Engineering (CSE) education. In view of this, theory and practice should be both covered [6]. Ideally, this should be done through Laboratory classes, whose main purpose should be to provide the connection between the abstract theory and the real world. These classes should also reinforce the idea that analytical approaches, theoretical and numerical, are useful in the sense that they can be accurately predictive. Physical intuition should also be stimulated towards promoting engineering judgement. Since many of the requirements of a control Laboratory, listed by Balchen et al [10], are difficult to fulfil, one solution could be to substitute expensive material by computer simulation [6]. The use of Matlab as a tool for solving and illustrate engineering problems has been advocated by Rossiter et al [11]. In a study conducted by Reck more recently is also clear the popularity of MathWorks software [13]. Moreover, Segway is a very good problem to integrate 
the use of computers as a way to visualisation in CSE, so advocated by S. Dormido et al [4], to promote intuition. Furthermore, a simpler version of the Segway can be build even by making the pieces in a $3 \mathrm{D}$ printer.

The inverted pendulum has been used in many instances, e.g. [5], to explain/illustrate simple concepts in control education, and also some research is still being conducted in this problem due to its many applications [12], [15]. According to Boubaker, it has been the most popular benchmark in CSE for more than fifty years, and in her work, she gives detailed explanation on how the inverted pendulum gives an effective and efficient application for learning control theory [7]. Furthermore, this has been used as a classical example to stabilise open-loop systems [8] and a typical root-locus analysis example [9]. Some established trends in robotics have also been based on the inverted pendulum stabilisation principle [7]. Kheir et al in [6] report the inverted pendulum as a very popular experiment in CSE education, and how it illustrates the difficulty to control an inherently unstable system. A tool for interactive learning based on this problem has been developed in [14]

In Section II, we linearise a model of the two-wheeled robot and calculate its respective transfer functions. In Section III, we analyse the system using techniques of classical control and assess its impulse response. We control the system using classical techniques. In Section IV, we propose a sequence of protocols based on the simplified Segway that cover all the course material of an introductory course in classical control as a way to implement it in the classroom.

In Section V, we withdraw some conclusions and outline a few directions that the work could be carried on.

\section{SEGWAY SIMPLIFIED MODEL}

The Segway considered in this work is a two-wheeled robot with a long rod mounted on the middle point of the wheels' axis; at the other extreme of the rod there is a mass. For simplicity sake of the model, the two wheels will be treated as a single unit and the robot travels along the $x$-axis. The rod is seen as a point mass rotating about the axis of the wheels. See Fig. 1. In order to have a model as simple as possible, we need a few additional assumptions [1]:

- the wheels are always in contact with the floor, rolling with no slip;

- the electrical and mechanical losses are considered to be zero;

- the dynamics of the electrical system is neglected, since the response of the electrical system is significantly faster than the one of the mechanical system;

- because the motion of the robot is constrained to a straight line, the system can be analysed as a 2-dimensional system;

- all bodies are rigid;

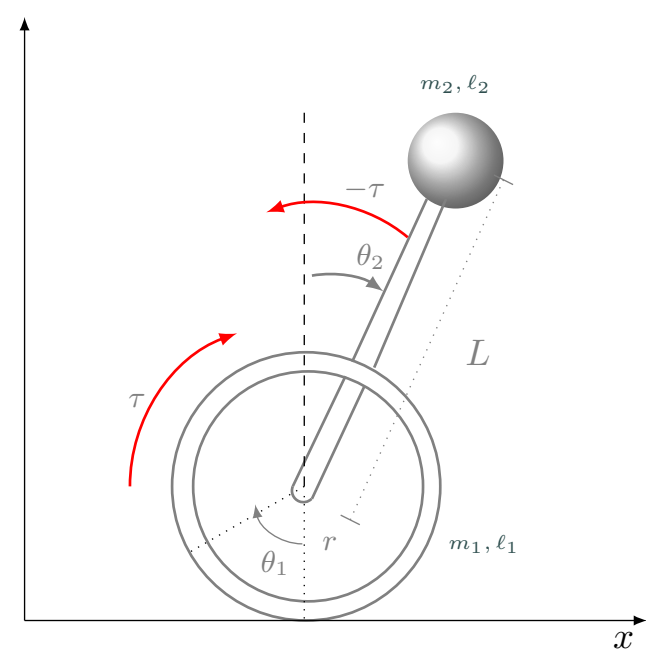

Fig. 1. The segway as an inverted pendulum in two-wheels.

- the angle of the tilt of the rod from the vertical should be sufficiently small to allow linearisation of the system $\left(\sin \left(\theta_{2}\right) \approx \theta_{2}\right)$

- to be able to neglect the centrifugal force, the angular velocity of the tilt is approximately zero $\left(\dot{\theta}_{2} \approx 0\right)$;

Under these assumptions, we consider the following nonlinear model without friction [2]:

$$
\begin{aligned}
\tau(t) & =H_{1} \ddot{\theta}_{1}(t)+H_{2}\left(\cos \left(\theta_{2}(t)\right) \ddot{\theta}_{2}(t)-\sin \left(\theta_{2}(t)\right) \dot{\theta}_{2}^{2}(t)\right), \\
-\tau(t) & =H_{2} \cos \left(\theta_{2}(t)\right) \ddot{\theta}_{1}(t)+H_{3} \ddot{\theta}_{2}(t)-a \sin \left(\theta_{2}(t)\right) .
\end{aligned}
$$

where:

$$
\begin{array}{ll}
H_{1}=\left(m_{1}+m_{2}\right) r^{2}+I_{1}, & H_{2}=m_{2} r L, \\
H_{3}=m_{2} L^{2}+I_{2}, & a=m_{2} g L .
\end{array}
$$

Also, $\tau(t)$ is the motor torque at every instant $t, m_{1}$ is the mass of the wheels, $m_{2}$ is the mass of the pendulum, $I_{1}, I_{2}$ are the robot and pendulum rotational moments of inertia, respectively, $r$ is the radius of the wheels, $\theta_{1}(t)$ is the angle of the position of the wheels and $\theta_{2}(t)$ is the rotation angle of the rod that we want to keep in the vertical position, both at every instant $t$. As the robot is meant to be kept in the vertical position, we use the two last assumptions mentioned above and the model is linearised. Also, we include in the model the frictional losses within the motor, therefore including in the torque its losses, i.e. $\tau(t)=\tau(t)+\tau_{\text {losses }}(t)$ with $\tau_{\text {losses }}(t)=b\left(\dot{\theta}_{1}(t)-\dot{\theta}_{2}(t)\right)$. We end up with the following simpler model:

$$
\begin{aligned}
\tau(t)-b\left(\dot{\theta}_{1}(t)-\dot{\theta}_{2}(t)\right) & =H_{1} \ddot{\theta}_{1}(t)+H_{2} \ddot{\theta}_{2}(t) \\
-\tau(t)+b\left(\dot{\theta}_{1}(t)-\dot{\theta}_{2}(t)\right) & =H_{2} \ddot{\theta}_{1}(t)+H_{3} \ddot{\theta}_{2}(t)-a \theta_{2}(t) .
\end{aligned}
$$




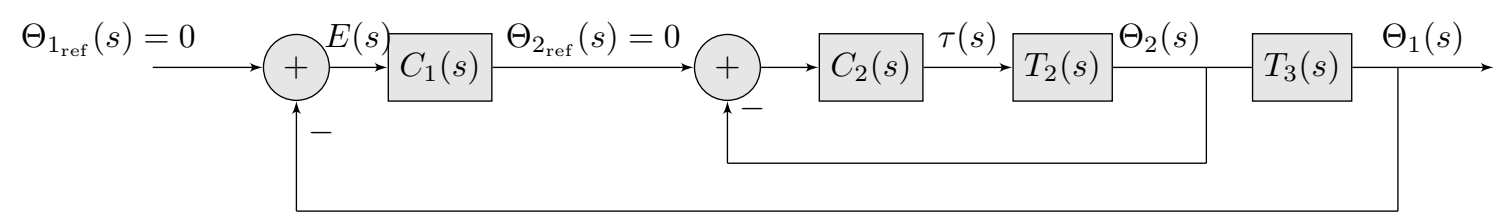

Fig. 2. Cascade feedback control of a SIMO system.

Defining $\quad \mathcal{H}:=\left[\begin{array}{ll}H_{1} & H_{2} \\ H_{2} & H_{3}\end{array}\right], \quad \mathcal{B}:=\left[\begin{array}{cc}b & -b \\ -b & b\end{array}\right]$ $\mathcal{K}:=\left[\begin{array}{cc}0 & 0 \\ 0 & -a\end{array}\right]$, we have:

$$
\left[\begin{array}{r}
\tau(t) \\
-\tau(t)
\end{array}\right]=\mathcal{H}\left[\begin{array}{l}
\ddot{\theta}_{1}(t) \\
\ddot{\theta}_{2}(t)
\end{array}\right]+\mathcal{B}\left[\begin{array}{l}
\dot{\theta}_{1}(t) \\
\dot{\theta}_{2}(t)
\end{array}\right]+\mathcal{K}\left[\begin{array}{l}
\theta_{1}(t) \\
\theta_{2}(t)
\end{array}\right]
$$

and after applying the Laplace Transform it becomes:

$$
\left[\begin{array}{c}
\tau(s) \\
-\tau(s)
\end{array}\right]=\left[\begin{array}{cc}
s^{2} H_{1}+b s & s^{2} H_{2}-b s \\
s^{2} H_{2}-b s & s^{2} H_{3}+b s-a
\end{array}\right]\left[\begin{array}{c}
\Theta_{1}(s) \\
\Theta_{2}(s)
\end{array}\right] .
$$

Furthermore, we define $D:=H_{1} H_{3}-H_{2}^{2}$ and $F:=H_{2}+H_{3}$ to obtain the following transfer functions:

$$
\begin{aligned}
& \tilde{T}_{1}(s)=\frac{\Theta_{1}(s)}{\tau(s)}=\frac{\left(H_{2}+H_{3}\right) s^{2}-a}{D s^{4}+b F s^{3}-a H_{1} s^{2}-a b s}, \\
& T_{2}(s)=\frac{\Theta_{2}(s)}{\tau(s)}=\frac{-\left(H_{1}+H_{2}\right) s}{D s^{3}+b F s^{2}-a H_{1} s-a b}, \\
& T_{3}(s)=\frac{\Theta_{1}(s)}{\Theta_{2}(s)}=\frac{-\left(H_{2}+H_{3}\right) s^{2}+a}{\left(H_{1}+H_{2}\right) s^{2}}
\end{aligned}
$$

We can also define $\omega(t)=\dot{\theta}_{1}(t)$, and consequently obtain $T_{1}(s)=s \tilde{T}_{1}(s)=\frac{\Omega(s)}{\tau(s)}$ where $\Omega(s)=\mathcal{L}(\omega(t))$, since in this way the pole at the origin of $s \tilde{T}_{1}(s)$ is cancelled.

\section{ANALYSIS AND CONTROL OF THE SYSTEM}

As the robot should be stopped and the pendulum kept upright, the output is the velocity of the wheels and the tilt of the angle of the rod. The control of the simplified Segway

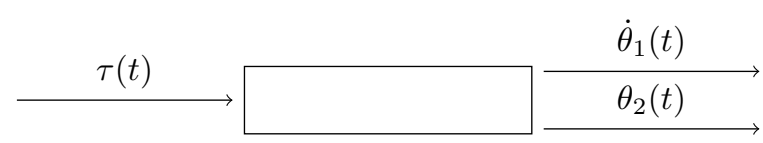

Fig. 3. Black-box representation of the SIMO system.

is clearly a SIMO system. However as we would like to resolve it using tools of classical control, which are clearly used for SISO systems, we use a successive loop closure approach (or cascade) in order to control the system. The idea is to define a feedback loop inside other feedback loop; the inner loop represents the control of the fast dynamics of the system, $\theta_{2}(t)$, while the outer loop represents the control of the slower dynamics, $\theta_{1}(t)$.
See Fig. 2. Therefore, we control $\theta_{2}(t)$ and analyse the effect of the controller on the robot position, i.e., on $\theta_{1}(t)$.

Using the following values for the parameters: $m_{1}=2 \mathrm{~kg}$, $m_{2}=4.5 \mathrm{~kg}, I_{1}=0.0032 \mathrm{kgm}^{2}, I_{2}=0.0065 \mathrm{kgm}^{2}, L=0.3 \mathrm{~m}$, $r=0.35 \mathrm{~m}, b=0.001 \mathrm{Nm} / \mathrm{rad} / \mathrm{s}$, transfer functions (9)-(11) become:

$$
\begin{aligned}
& T_{1}(s)=\frac{8.3619\left(s^{2}-3.869^{2}\right)}{(s+10.01)(s-9.993)(s+0.001251)}, \\
& T_{2}(s)=\frac{-12.032 s}{(s+10.01)(s-9.993)(s+0.001251)}, \\
& T_{3}(s)=\frac{-0.695\left(s^{2}-3.869^{2}\right)}{s^{2}} .
\end{aligned}
$$

Our first concern is to stabilise the system, which means to stabilise the inner loop and at the same time obtain a feasible response for the outer loop. Additionally, we can consider other design criteria such as settling time or maximum overshoot.

To design controller $C_{2}(s)$, we start by plotting the rootlocus of $T_{2}(s)$, from which becomes clear that we need a controller with negative gain to stabilise the system; with $C_{2}=K_{2}, K_{2}>0$, a branch always exists on the right-half plane.
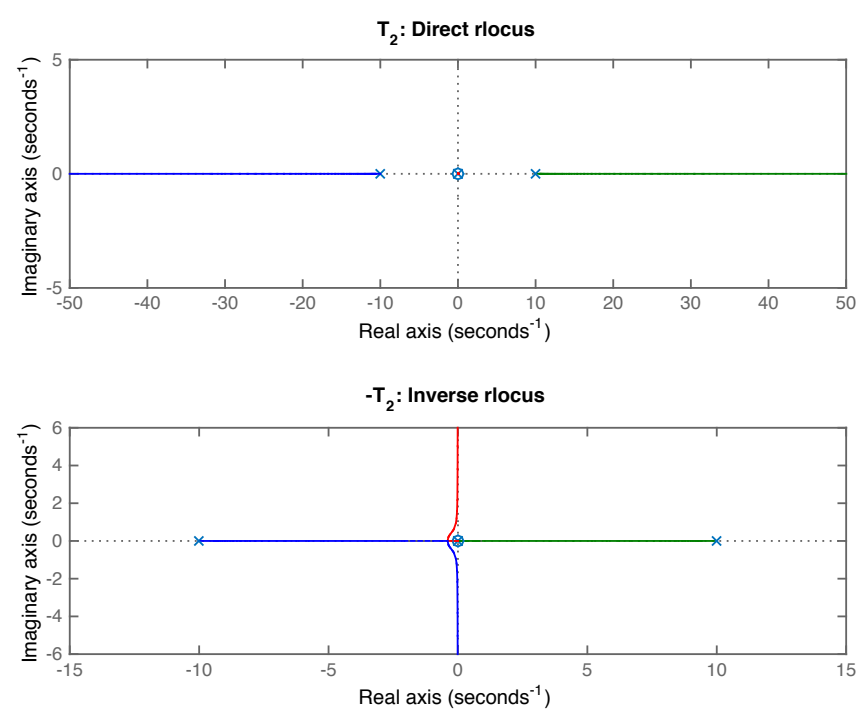
Fig. 4. $\quad T_{2}(s)=\frac{-12.032 s}{(s+10.01)(s-9.993)(s+0.001251)}:$ direct and
inverse rlocus. 
A PID approach, although being a very easy to apply technique, is not intuitive and the empirical tuning methodse.g. Ziegler-Nichols, Chen-Coon-have been designed only for stable systems. Therefore, what is left is an endless trial and error search of parameters. This well illustrates the limitations of the use of the proportional, integral and derivative actions to the students. The students should look for other type of compensators to stabilise $T_{2}(s)$. Paramount at this point is also to use different techniques of classical control to assess the response of the system for the different compensators obtained.

We tune the system from the root-locus of $-T_{2}(s)$. The rootlocus is a simple visual tool where the system "stability" is reliably represented and the changes made on the system by the controller are readily perceived. The drawback is the necessary number of iterations to tune the system to the required performance. Observing the lower plot of Fig. 4, one sees that the most left and the centred branches always remain within the left-half plane, but the the most right one is a direct connection between the positive pole and the zero at the origin, and thus never crosses the imaginary axis, that is, the system remains unstable with $C_{2}=K_{2}, K_{2}<0$. To force this branch to leave the real axis, another positive pole is added: $C_{2}=\frac{-K_{2}}{s-5}, K_{2}>0$. See the upper plot of Fig. 5 . However, although the root-locus branch leaves the real axis it drifts away from the left-half plane. To attract it back, a zero needs to be added: $C_{2}=\frac{-K_{2}(s+1)}{s-5}, K_{2}>0$. See the lower plot of Fig. 5.
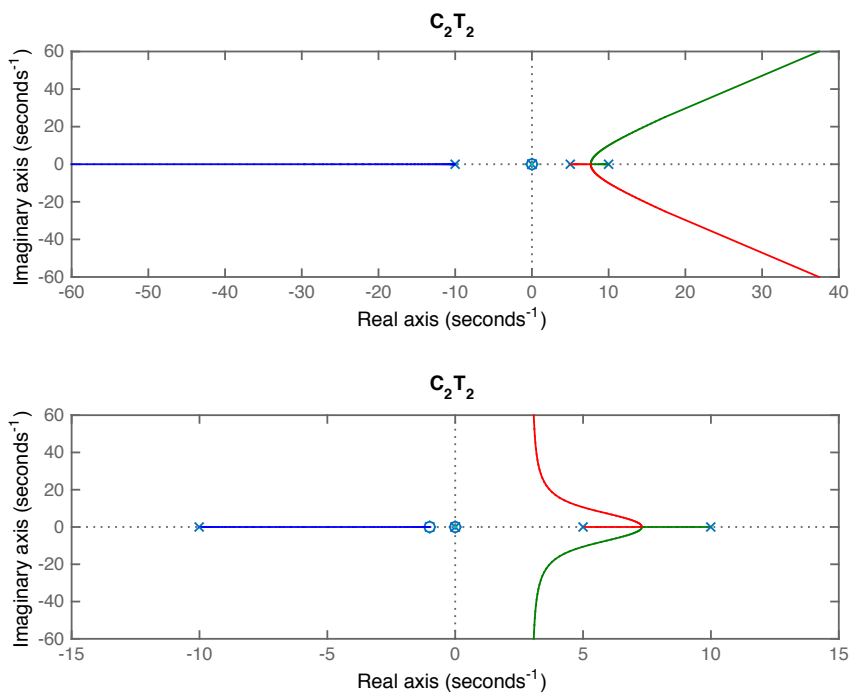

Fig. 5. Construction of $C_{2}$ for the fast dynamics closed-loop system.

Although the branch has been deviated to the left, it was not enough, and adding another zero is necessary. For the resulting transfer function to remain a proper rational function, another pole is also included. That is, to shift the root-locus of the bottom figure of Fig. 5 to the one of
Fig. 6, a lead compensator is added and we end up with $C_{2}=\frac{-K_{2}(s+1)(s+12)}{(s+40)(s-5)}, K_{2}>0$.

The upper plot of Fig. 6 shows the root-locus of $C_{2} T_{2}$, with $K_{2}=80$, and the resulting stability margins are shown in the lower plot. Thus, we obtain a closed-loop stable system with
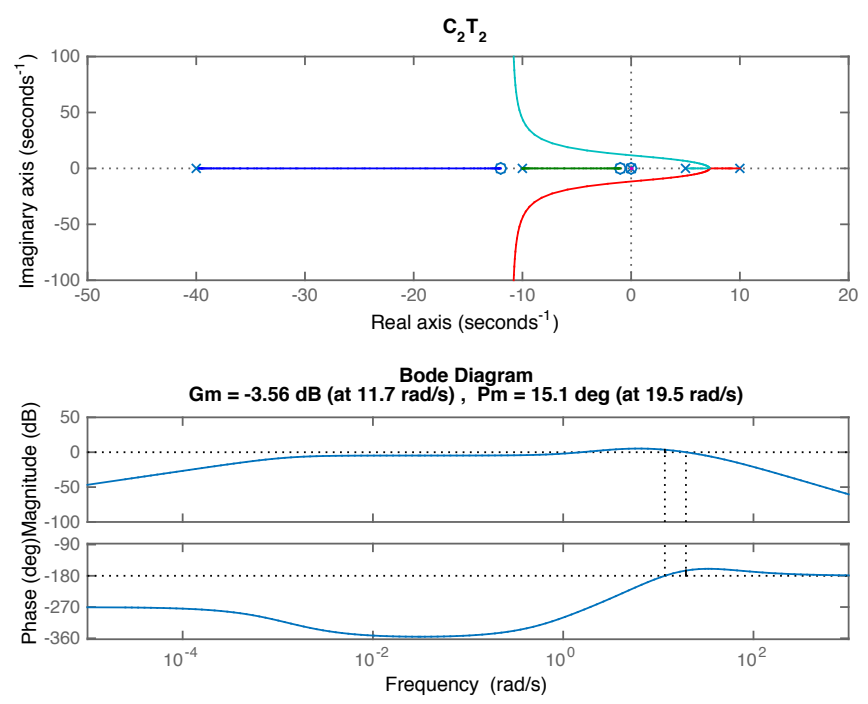

Fig. 6. Root-locus and frequency analysis of $C_{2} T_{2}$ for the closed-loop fast dynamics system with $C_{2}=\frac{-80(s+1)(s+12)}{(s+40)(s-5)}$.

three real negatives poles, although one of them is very close to the origin, and two complex poles with negative real part, and a reasonably small damping, therefore $\frac{\Theta_{2}(s)}{R_{2}(s)}$ becomes:

$\frac{962.53 s(s+12)(s+1)}{(s+20.45)(s+4.851)(s+0.0007934)\left(s^{2}+9.704 s+318\right)}$.

The impulse response of the closed loop $C_{2} T_{2}(s)$ system, and the resulting impulse response of $s T_{3}(s)$ are both shown in Fig. 7. The physical response of the latter is not feasible as the wheels start to move backwards.

As we have not been able to design a controller $C_{2}(s)$ that produces a satisfactory response for $\dot{\theta}_{1}(t)$, from the physical point of view, our next task is to design a controller, $C_{1}$, for the outer loop to produce the desired behaviour. Although the cancellation of poles and zeros is usually considered a poor design due to drifting, in this situation we find it acceptable since the losses are mainly drifting and viscous forces. Therefore, we chose a controller with negative gain and that cancels the positive zero of $T_{3}(s): C_{1}=\frac{-K_{1}}{s-3.869}, K_{1}>0$. The behaviour of the closed loop controlled system is described in Fig. 8 and Fig. 9. 

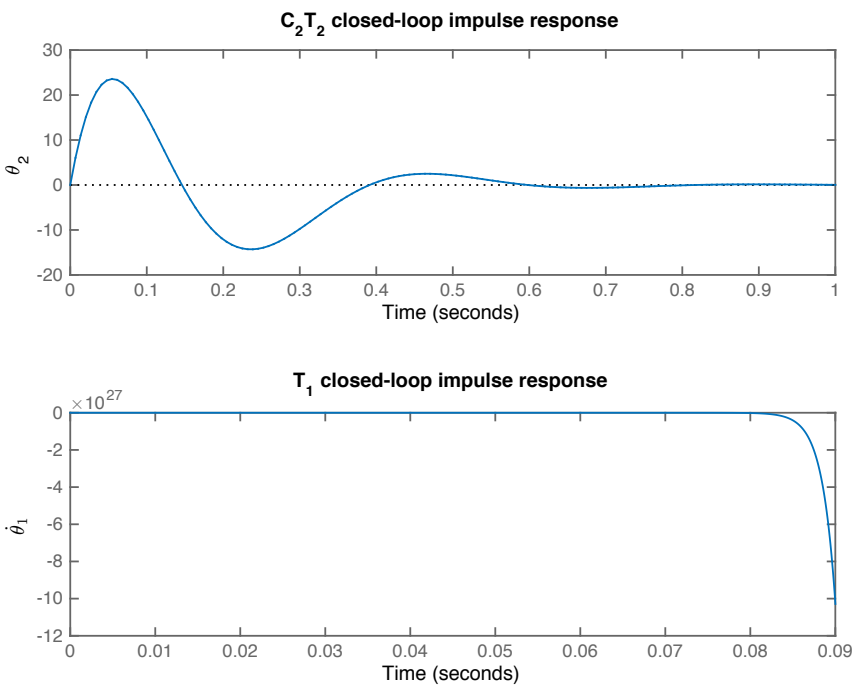

Fig. 7. Analysis of the feasibility of the system after designing the contro of the closed-loop fast dynamics system. $C_{2}=\frac{-80(s+1)(s+12)}{(s+40)(s-5)}$.
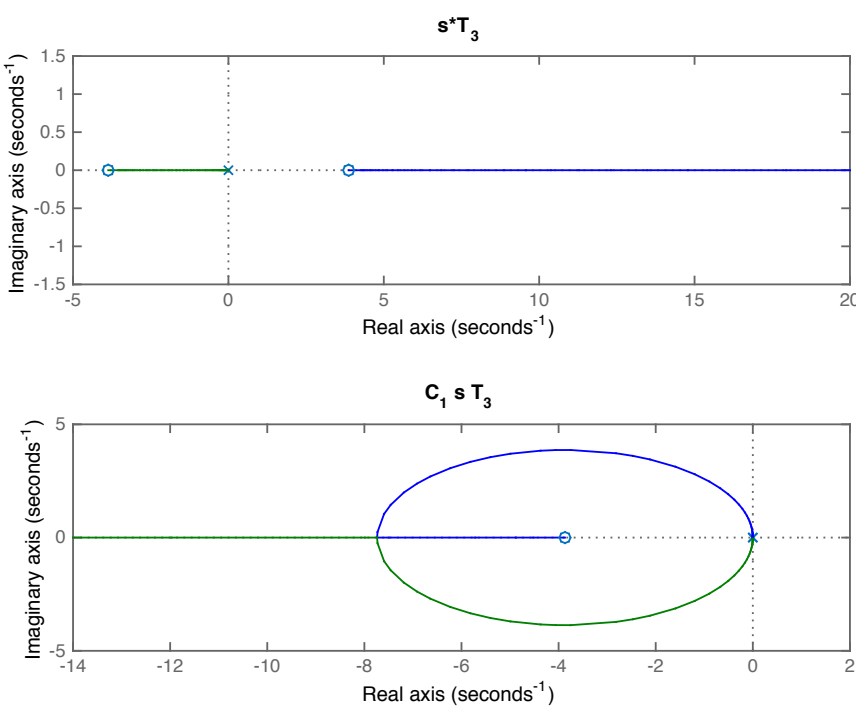

Fig. 8. Analysis of the effect of controller $C_{1}=\frac{-1.5}{s-3.869}$ on the root-locus of $s T_{3}(s)=\frac{-0.695\left(s^{2}-3.869^{2}\right)}{s^{2}}$.

\section{THE SEgWAY TAKEN TO THE CLASSROOM}

In this section we propose a series of four protocols to integrate the material of a course of classical control in the analysis and control of the two-wheeled robot. By using Matlab to do the simulations, we also integrate computing into the work [6]. The same analysis can be done also with Simulink.

In the protocol 1:

- To linearise model (1)-(2) and calculate the transfer functions.

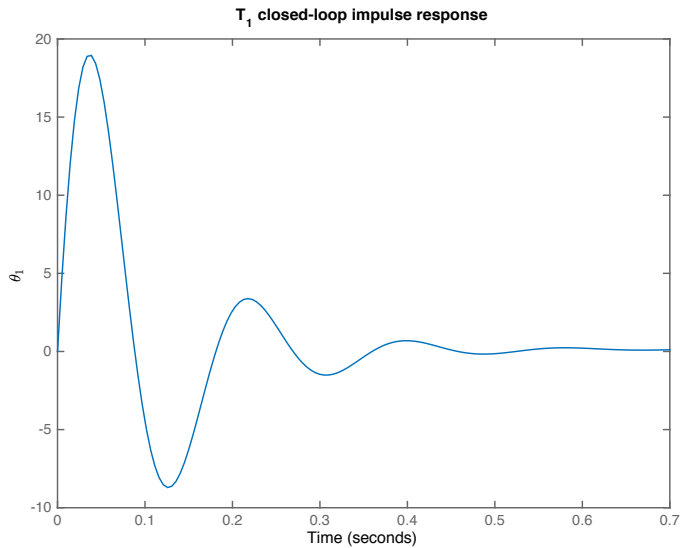

Fig. 9. Impulse response of the slow dynamics closed-loop system with $C_{1}=\frac{-1.5}{s-3.869}$

- To calculate the gain, poles and zeros of the transfer functions, and understand its meaning in the real problem.

- To understand the meaning of impulse response and learn how to simulate it.

- To distinguish between natural response, forced response and free response. To learn how to simulate everyone of these responses.

In the protocol 2:

- To distinguish between open-loop and close-loop response.

- To understand the Segway as an intrinsically unstable system.

- To understand block diagrams and use them to define the control strategy.

- Analyse the limitations of a PID controller to stabilise the system.

- To verify the physical feasibility of the whole system.

In the protocol 3:

- To learn how to sketch the root-locus, even with negative gain.

- To design a compensator with the help of the root-locus to stabilise the inner closed-loop system.

- To use frequency response tools to assess the performance of the controlled system.

- To understand the meaning of step response and learn how to simulate it.

- To analyse the resulting steady-state error.

- To design a compensator according to some other system specification, for instance damping, using the second order system approximation.

In the protocol 4:

- To analyse the response of the outer loop: $\dot{\theta}_{1}$. 
- To design a controller to stabilise the outer loop system of the diagram depicted in Fig. 2.

- To evaluate the feasibility of the whole system.

- To study the same problem with non zero initial conditions for $\theta_{2}(0)$.

\section{CONCLUSION AND DIRECTIONS FOR FUTURE WORK}

In this work, we analysed the use of a simplified Segway model to integrate theory with practice in teaching/learning of control, towards to the development of intuition and engineering judgment. This is a nonlinear SIMO system.

In view of the stabilisation of a SIMO system using techniques of classical control, a cascade control strategy has been used, where the inner feedback loop is first stabilised, followed by the stabilisation of the slower dynamics of the system. The control and analysis of the system is structured in a set of four protocols, that are meant to be implement in the classroom through Matlab simulation.

The outlined protocols comprise two different control problems: (i) a simpler one, that is to swing the pendulum from the equilibrium position and then return it to the upright position. (ii) a more general one, that is to return the pendulum from any initial condition $\left(\theta_{2}(0)=\theta_{2}\right)$ to the upright one and then stabilise the robot in the desired position.

The problem under discussion in this work can be used to illustrate every topic of a classic control theory course, and also make the transition to the state-space techniques, where the multi-variable controller can be calculated in a more direct manner.

Also, we would like to point out that this is not a very trivial problem and raises many relevant questions in learning of control theory. However, whenever the problem might be found too complex, it may substituted by a simpler version, that is, in equations (5)-(6) the losses term of the motor could be dropped. When the friction term is not included, the analysis and control of the whole problem becomes much easier.

In future work, we would like to propose to the students some analysis of the same problem using Simulink, and considering different specifications of the problem as for instance the robot being not stopped (and with a constant speed). Furthermore, as the design of the controller for the outer feedback loop is still an open problem, we would like to study the same problem using state-space control techniques. State-space techniques can also be used to solve the linear optimal control problem for unstable systems. The work of the Laboratory would follow the same structure proposed here.

Therefore, although being a small problem, the simplified Segway is a very fruitful one from the Control Systems Engineering education point of view.

\section{ACKNOWLEDGMENT}

The authors would like to thank the reviewers for their comments that helped in improving this document.

The authors acknowledge Fundação para a Ciência e Tecnologia (FCT) and COMPETE 2020 program for the financial support to the project UID-EEA-00048-2013.

\section{REFERENCES}

[1] Bageant, M.R., Balacing a two-wheel segway robot; BSc Thesis, MIT, USA, June, 2011

[2] Castro, A., Modelling and dynamic analysis of a a two-wheeled inverted; MSc Thesis, Georgia Institute of Technology, USA, August, 2012.

[3] Aström, J. K., Challenges in Control Education; Department of Automatic Control, Lund University, Madrid, 2006.

[4] Dormido, S., S.Dormido-Canto, R.Dormido and J.Sánchez, The role of interactivity in control learning; International Journal of Engineering Education, vol 21(6), pp 1122-1133, 2005.

[5] Sánchez, J., S.Dormido, R.Pastor and F.Morilla, A Java/Matlab-based environment for remote control system laboratories: Illustrated with an inverted pendulum; IEEE Transactions on Education, vol 47(3), pp 321329, 2004.

[6] Kheir, N.A., K.J.Aström, D.Auslander, K.C.Cheok, G.F.Franklin, M.Masten and M.Rabins, Control systems engineering education; $A u-$ tomatica, vol 32(2), pp 147-166, 1996.

[7] Boubaker, O., The inverted Pendulum: A fundamental Benchmark in Control Theory and Robotic; IEEE International Conference on Education and e-Learning Innovations (ICEELI), Sousse, Tunisia, 1-3 July, 2012.

[8] Kailath,T., Linear Systems. Englewood Cliffs: Prentice-Hall, 1980.

[9] Siebert, W. McC., Circuits, Signals, and Systems. Cambridge: MIT Press, 1998.

[10] Balchen, J. G., M. Handlykken and A. Tyss, The need for better laboratory experiments in control engineering education; In Proc. 8th IFAC Triennial World Congress, Kyoto, Japan, 1981.

[11] Rossiter, J.A. and L. Gray, L, Supporting development of independent learning skills; 2010 IEEE Education Engineering, Madrid, Spain, 14 16 April, 2010.

[12] H. Lee and J. Lee, Driving Control of Mobile Inverted Pendulum; The 9th International Conference on Ubiquitous Robots and Ambient Intelligence (URAI 2012), Daejeon, Korea, November 26-28, 2012.

[13] Reck, R.M., Defining Common Aspects of Undergraduate Instructional Laboratories for Control Systems; American Control Conference (ACC), Boston,MA, USA, July 6-8, 2016.

[14] Lima, J.L., J.C. Gonçalves, P.G.Costa and A.P.Moreira, Inverted pendulum virtual control laboratory. Incomplete reference.

[15] Kafetzis,I. and L.Moysis, Inverted Pendulum: A system with innumerable applications; School of Mathematical Sciences, Aristotle University of Thessaloniki, Thessaloniki, Greece. 